\title{
An inducible mouse model for skin cancer reveals distinct roles for gain- and loss-of-function p53 mutations
}

\author{
Carlos Caulin, ${ }^{1}$ Thao Nguyen, ${ }^{1}$ Gene A. Lang, ${ }^{2}$ Thea M. Goepfert, ${ }^{3}$ Bill R. Brinkley, ${ }^{3}$ \\ Wei-Wen Cai, ${ }^{4}$ Guillermina Lozano, ${ }^{2}$ and Dennis R. Roop ${ }^{1,3}$ \\ ${ }^{1}$ Department of Dermatology, Baylor College of Medicine, Houston, Texas, USA. ²Department of Molecular Genetics, \\ University of Texas MD Anderson Cancer Center, Houston, Texas, USA. ${ }^{3}$ Department of Molecular and Cellular Biology and \\ ${ }^{4}$ Department of Molecular and Human Genetics, Baylor College of Medicine, Houston, Texas, USA.
}

\begin{abstract}
Mutations in ras and p53 are the most prevalent mutations found in human nonmelanoma skin cancers. Although some $p 53$ mutations cause a loss of function, most result in expression of altered forms of $\mathrm{p} 53$, which may exhibit gain-of-function properties. Therefore, understanding the consequences of acquiring $p 53$ gainof-function versus loss-of-function mutations is critical for the generation of effective therapies for tumors harboring $p 53$ mutations. Here we describe an inducible mouse model in which skin tumor formation is initiated by activation of an endogenous $K-r^{G} s^{G 12 D}$ allele. Using this model we compared the consequences of activating the $p 53$ gain-of-function mutation $p 53^{R 172 H}$ and of deleting the $p 53$ gene. Activation of the $p 53^{R 172 H}$ allele resulted in increased skin tumor formation, accelerated tumor progression, and induction of metastasis compared with deletion of $p 53$. Consistent with these observations, the $p 53^{R 172 H}$ tumors exhibited aneuploidy associated with centrosome amplification, which may underlie the mechanism by which $p 53^{R 172 H}$ exerts its oncogenic properties. These results clearly demonstrate that $p 53$ gain-of-function mutations confer poorer prognosis than loss of $p 53$ during skin carcinogenesis and have important implications for the future design of therapies for tumors that exhibit $p 53$ gain-of-function mutations.
\end{abstract}

\section{Introduction}

Nonmelanoma skin cancer (NMSC) is the most common form of human cancer, with incidence rates dramatically rising during the last decade, perhaps as a result of increased sun exposure and the continuous depletion of the ozone layer $(1,2)$. It is widely accepted that squamous cell carcinomas (SCCs), which are responsible for the majority of NMSC-related deaths, result from the accumulation of genetic alterations (3). Understanding the role of these genetic lesions in the etiology of skin cancer is essential for designing improved therapies for cancer treatment and prevention.

Mutations in ras genes are found in approximately $10 \%-30 \%$ of human skin SCCs $(4,5)$, and mice subjected to chemical carcinogenesis protocols by initiation with dimethylbenz[a]anthracene (DMBA) and tumor promotion with 12-O-tetradecanoylphorbol13 -acetate (TPA) develop skin tumors that exhibit $H$-ras mutations in more than $90 \%$ of the cases (6). In addition, mice treated with the carcinogen $N$-methyl- $N^{\prime}$-nitro- $N$-nitrosoguanidine and $H$-ras-null mice subjected to DMBA/TPA protocols develop skin tumors that carry oncogenic mutations in the K-ras gene, suggesting that mutations in $K$-ras can substitute for $H$-ras mutations in skin cancer initiation $(7,8)$. Several mouse models have previously documented that overexpression of oncogenic $H$-ras or $K$-ras in the skin can induce tumor formation (9-12). However, activation of ras genes associated with skin cancer initiation may not necessarily

Nonstandard abbreviations used: BAC, bacterial artificial chromosome; BAC-CGH, comparative genomic hybridization on BAC; DMBA, dimethylbenz[a]anthracene; K5, keratin 5; NMSC, nonmelanoma skin cancer; SCC, squamous cell carcinoma; TPA, 12-O-tetradecanoylphorbol-13-acetate.

Conflict of interest: The authors have declared that no conflict of interest exists. Citation for this article: J. Clin. Invest. 117:1893-1901 (2007). doi:10.1172/JCI31721. result in overexpression of the mutant ras allele, suggesting that mutation of endogenous ras in the mouse may better reproduce the initial event in human skin carcinogenesis.

More than $50 \%$ of human cutaneous SCCs carry mutations in the $p 53$ gene, most of which are missense mutations that result in the change of a single amino acid and expression of altered forms of $\mathrm{p} 53(13,14)$. Some of these $p 53$ mutations can promote tumorigenicity when introduced into $p 53$-null cells, suggesting that they acquire gain-of-function properties $(15,16)$. Several lines of evidence, including clinical observations, suggest that tumors containing putative $p 53$ gain-of-function mutations may have a poorer prognosis than those with loss of p53 (17). In addition, 2 recently generated knockin mouse models provided evidence to suggest that mutant p53 may also exhibit gain-of-function properties in vivo $(18,19)$. We previously generated a transgenic mouse model that overexpressed $p 53^{R 172 H}$ (equivalent to human $p 53^{R 175 H}$ ) in the epidermis and found that these mice exhibited accelerated chemical carcinogenesis (20). Since the $p 53^{R 172 H}$ transgene was expressed at levels 4- to 8-fold higher than WT $p 53$, it was unclear whether $p 53^{R 172 H}$ functioned as a true gain-of-function mutation in the genetic sense.

Here we describe the generation of an inducible mouse model for skin cancer based on the focal activation of an endogenous K-ras ${ }^{G 12 D}$ allele and gain- or loss-of-function mutations in $p 53$. This model allowed us to compare the consequences of activating endogenous p53 gain-of-function versus loss-of-function mutations during skin cancer development. To our knowledge, this is the first study in which these mutations have been compared in a tissue-specific manner with a common initiating event $\left(K-\right.$ ras $\left.^{G 12 D}\right)$. In this context, the mutant $p 53^{R 172 H}$ allele clearly exhibits gain-of-function properties that contribute to skin cancer initiation and malignant progression. 

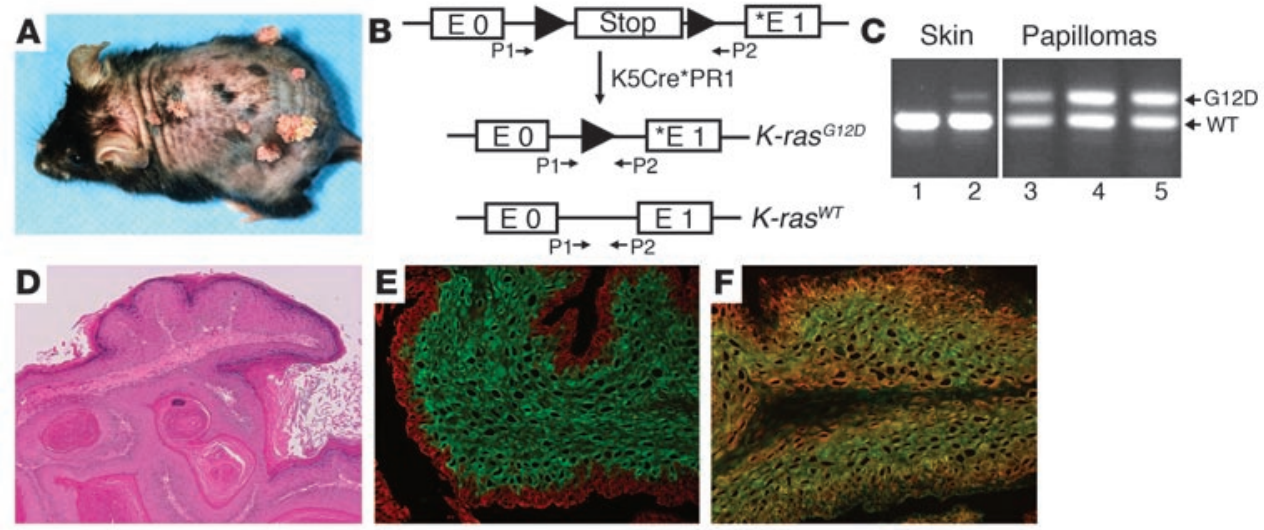

\section{Figure 1}

K-ras activation initiates skin tumor formation. (A) Gross appearance of the epidermal papillomas that developed in the skin of RU486-treated K5Cre*-Ras mice 16 weeks after exposure to TPA. (B) Schematic representation of the $L S L-K$-ras ${ }^{G 12 D}$ allele. The glycine-to-aspartic acid mutation (asterisk) in codon 12 is located in exon 1 (E1). Primers 1 and 2 (P1 and P2) were used for analysis of excision of the stop cassette. (C) Activation of the $K$-ras $^{G 12 D}$ allele in the skin (lanes 1 and 2) and papillomas (lanes 3, 4, and 5) of K5Cre*-Ras mice. Note that the $K$-ras ${ }^{G 12 D}$ allele is only activated in RU486-treated skin (lane 2), but not in untreated mice (lane 1). (D) Hematoxylin and eosin staining of papillomas that developed in $\mathrm{K} 5 \mathrm{Cre}$-Ras mice. (E and F) Keratin staining in papillomas: double immunofluorescence for K14 (red) and K13 (green) (E) and K14 (red) and K6 (green) (F) on frozen sections obtained from papillomas that developed in K5Cre*-Ras mice. Original magnification, $\times 40$ (D), x100 (E and F).

\section{Results}

Activation of an endogenous $K$-ras ${ }^{G 12 D}$ mutation initiates skin tumor formation. To activate an endogenous oncogenic ras mutation in mouse skin, as occurs in human sporadic skin cancer, we generated mice in which the K-ras ${ }^{G 12 D}$ mutation could be focally induced in the skin by crossing LSL-K-ras ${ }^{\mathrm{G} 12 \mathrm{D}}$ mice with K5.Cre*PR1 mice (K5Cre*Ras mice) $(21,22)$. The LSL-K-ras ${ }^{\mathrm{G} 12 \mathrm{D}}$ mice carry an endogenous $K$-ras allele that contains the G12D mutation, a mutation frequently found in human tumors, and a stop cassette flanked by loxP sites (LSL) that keeps the $L S L-K$-ras ${ }^{G 12 D}$ allele silent until the stop cassette is deleted by Cre recombinase (Figure 1B). The K5.Cre* PR1 mice express an inducible Cre recombinase under the control of the keratin 5 (K5) promoter. In these mice the inducible Cre is expressed in stratified epithelia including the skin and remains inactive until activation with RU486. Topical application of RU486 to the skin of $\mathrm{K} 5 \mathrm{Cre}{ }^{*}$-Ras mice activates Cre in the epidermis, which results in deletion of the stop cassette from the $L S L-K$-ras ${ }^{G 12 D}$ allele and expression of endogenous $K-$ ras $^{G 12 D}$ (Figure 1B).

To determine whether endogenous activation of $K$-ras ${ }^{G 12 D}$ can initiate skin tumor formation, we treated $\mathrm{K} 5 \mathrm{Cre}{ }^{*}$-Ras mice with a single topical application of RU486 $(1 \mathrm{mg} / \mathrm{ml})$ and weekly applications of the tumor promoter TPA. We found that $\mathrm{K} 5 \mathrm{Cr} \mathrm{e}^{*}$-Ras mice developed skin tumors 7-10 weeks after tumor promotion (Figure 1A). Mice that were not treated with RU486 did not develop skin tumors, indicating that the inducible system was tightly regulated in vivo. PCR analysis with primers that flank the stop cassette in the $L S L-K-r a s^{G 12 D}$ allele, using DNA purified from the skin of mice that were treated with RU486 and untreated mice, confirmed that the K-ras ${ }^{G 12 D}$ allele was only activated in the RU486-treated skin, proving that activation of conditional alleles in this model only occurred after treatment with RU486 (Figure 1, B and C). The tumors that developed in $\mathrm{K} 5 \mathrm{Cr}{ }^{*}$-Ras mice exhibited similar levels of the activated mutant $K-$ ras $^{G 12 D}$ and WT alleles, indicating that the tumors consisted primarily of epithelial cells that activated the mutant $K$-ras ${ }^{G 12 D}$ allele (Figure $1 C$ ). The skin tumors that arose in the $\mathrm{K} 5 \mathrm{Cre}$-Ras mice were benign papillomas histologically similar to those generated in classic 2-stage chemical carcinogenesis protocols using DMBA/TPA (Figure 1D). Analysis of keratin expression revealed that the $\mathrm{K} 5 \mathrm{Cr} \mathrm{C}^{*}$-Ras papillomas expressed $\mathrm{K} 14$ in the entire epithelial component of the tumors, K13 in the most differentiated layers of the papillomas, and K6 in all the epithelial layers of the tumor (Figure 1, E and F), a keratin expression pattern similar to that observed in chemically induced papillomas $(23,24)$. Overall, these results demonstrate that activation of an endogenous oncogenic ras allele can initiate skin tumor formation. These results also document that the inducible Cre mice are ideal for generating mouse models for skin cancer and for testing the cooperativity of discrete genetic alterations detected in sporadic human cancers.

The $p 53^{R 172 H}$ gain-of-function mutation contributes to skin cancer initiation and accelerates carcinoma formation. To determine whether endogenous ras and p53 mutations cooperate during skin carcinogenesis, we used the inducible system to generate mice in which endogenous ras and p53 mutations could be activated in the skin. Since most $p 53$ mutations that occur in human tumors, including NMSC, are missense mutations that may exhibit gain-of-function properties (25), it was important to determine whether endogenous $p 53$ gain-of-function and loss-of-function mutations confer different skin cancer susceptibility. To this end, we generated mice in which the $K-r a s^{G 12 D}$ and $p 53^{R 172 H}$ alleles could be activated in the skin upon treatment with RU486 (K-ras-p53 R172H/WT mice), by crossing $\mathrm{K} 5 \mathrm{Cre}{ }^{*}$-Ras mice with Neo-p $53^{\mathrm{R} 172 \mathrm{H}}$ mice, which carry a conditional $p 53^{R 172 H}$ allele (18) (Figure $2 \mathrm{~A}$ ). To compare the effects of the $p 53^{R 172 H}$ gain-of-function mutation with loss of $p 53$, we also generated mice in which the $K$-ras ${ }^{G 12 D}$ mutation and deletion of $p 53$ could be induced in the skin (K-ras-p53/WT mice) by crossing the $\mathrm{K} 5 \mathrm{Cre}{ }^{*}$-Ras mice with floxed $p 53$ mice ( $\left.p 53^{f}\right)$ (26) (Figure $2 \mathrm{~B})$. The breeding strategy followed to generate these mice allowed us to compare K-ras-p53 $3^{\mathrm{R} 172 \mathrm{H} / \mathrm{WT}}$ and K-ras-p53 ${ }^{\mathrm{f} / \mathrm{WT}}$ littermates, thereby minimizing genetic background effects. We found that after treatment with TPA, the K-ras-p53 $3^{\mathrm{R} 172 \mathrm{H} / \mathrm{WT}}$ mice developed 
A
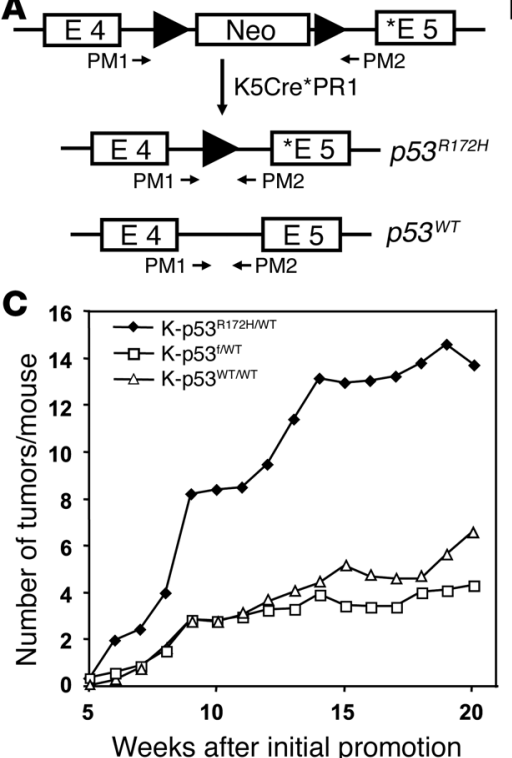

E

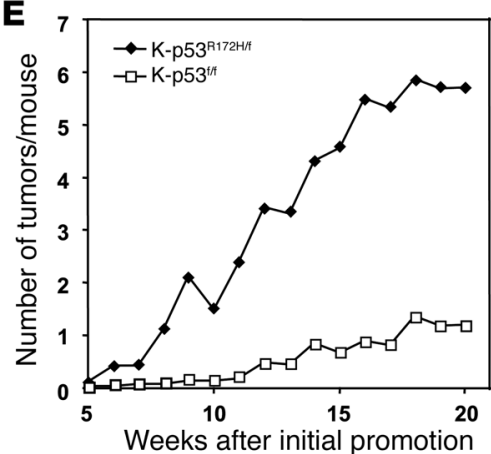

G
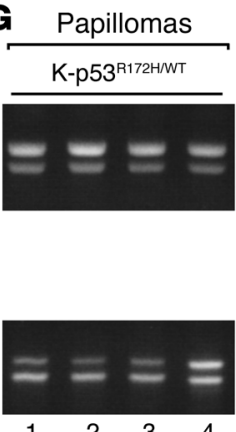

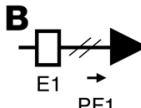

E2Е3 E4 E5 E6 E7 E8 E9 E10 K5Cre*PR1
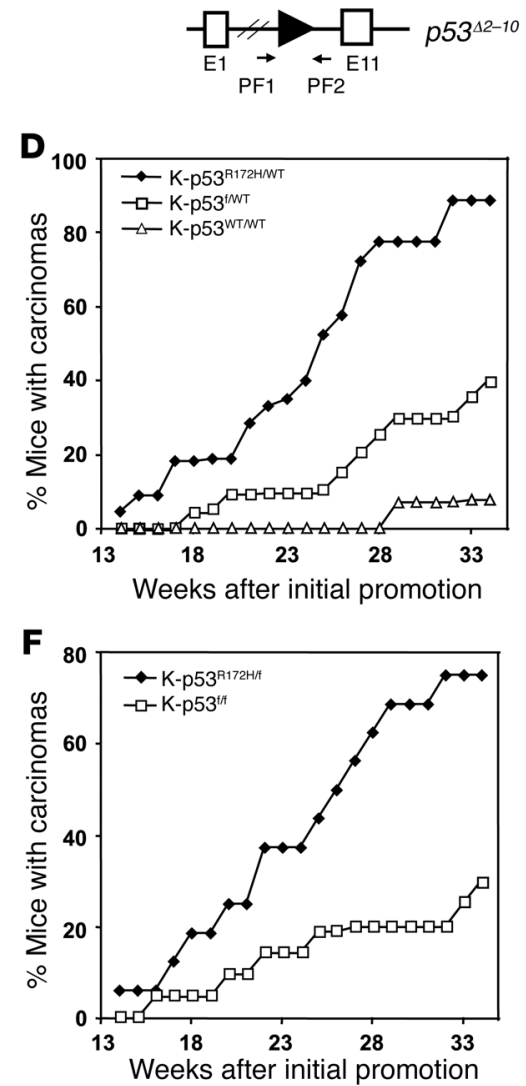

Figure 2

Activation of the $p 53^{R 172 H}$ allele contributes to tumor initiation and malignant progression of skin tumors. (A) Schematic representation of the neo-p53 ${ }^{R 172 H}$ allele showing exons 4 and 5 , the presence of the neo cassette in intron 4, and primers $\mathrm{R} 172 \mathrm{H}$ reverse and $\mathrm{R} 172 \mathrm{H}$ forward (PM1 and PM2, respectively; see Methods), which were used for PCR analysis of the $p 53$ allele. The neo cassette prevents expression of the $p 53^{R 172 H}$ allele (C. Caulin, D.R. Roop, and G. Lozano, unpublished observations). Asterisks indicate mutation in exon 5 for $\mathrm{p} 53^{\mathrm{R} 172 \mathrm{H}}$.

(B) Schematic representation of the $p 53^{f}$ allele showing loxP sites in introns 1 and 10 and primers PF1 and PF2 (26) that were used to analyze deletion of exons 2-10. (C) Kinetics of tumor formation in K-ras-p53 ${ }^{\mathrm{R} 172 \mathrm{H} / \mathrm{WT}}$ mice (K-p53R172H/WT; $n=22$ ) and K-ras-p53/WT mice (K-p53 $\left.{ }^{\mathrm{f} / \mathrm{WT}} ; n=21\right)$. K-ras-p53 ${ }^{\mathrm{WT} / \mathrm{WT}}$ mice (K-p53 ${ }^{\text {WT/WT; }} n=17$ ) were used as controls. Tumor formation was evaluated by the average number of tumors developed per mouse after the initial treatment with TPA. (D) Kinetics of carcinoma formation. Each time point represents percentage of mice bearing carcinomas. (E) Kinetics of tumor formation in K-ras-

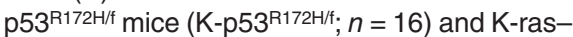
$\mathrm{p} 53^{\mathrm{f} / \mathrm{f}}$ mice (K-p53 $\left.\mathrm{ft/f} ; n=22\right)$. (F) Kinetics of carcinoma formation in K-ras-p53 $172 \mathrm{H} / \mathrm{f}$ and K-rasp53/f/ mice. (G) Activation of the p53 ${ }^{R 172 H}, p 53^{f}$, and $K$-ras ${ }^{G 12 D}$ alleles in papillomas and carcinomas. Primers PM1 and PM2 were used to analyze activation of the $p 53^{R 172 H}$ allele. Primers PF1 and PF2 were used to analyze deletion of the loxP-flanked sequences in the $p 53^{f}$ allele. Primers P1 and P2 were used to analyze activation of the $K$-ras ${ }^{G 12 D}$ allele. DNA purified from spleen (Sp; lane 16) of a K-ras-p53 ${ }^{\text {R172H/WT }}$ mouse was used as a control and only generated the band corresponding to $p 53^{W T}$ or $K$-ras ${ }^{W T}$ alleles.
3 -fold more tumors than K-ras-p53/ $/ \mathrm{WT}$ mice and mice carrying 2 WT $p 53$ alleles (K-ras-p53 WT/WT) (Figure 2C). These results indicate that the gain-of-function $p 53^{R 172 H}$ mutation, but not a $p 53$ loss-offunction mutation, cooperates with $K$-ras ${ }^{G 12 D}$ in skin cancer initiation. In addition, we found that carcinoma development was dramatically accelerated in K-ras-p $53^{\mathrm{R} 172 \mathrm{H} / \mathrm{WT}}$ mice compared with K-ras-p53 ${ }^{\mathrm{f} / \mathrm{WT}}$ and K-ras-p53 ${ }^{\mathrm{WT} / \mathrm{WT}}$ mice (Figure 2D). Thus, by 32 weeks, $90 \%$ of the K-ras-p53 ${ }^{\mathrm{R} 172 \mathrm{H} / \mathrm{WT}}$ mice had developed skin carcinomas compared to $5 \%$ of K-ras-p53 $3^{\mathrm{WT} / \mathrm{WT}}$ littermates and $30 \%$ of the K-ras-p53 $3^{\mathrm{f} / \mathrm{WT}}$ mice. These data suggest that expression of an endogenous $p 53^{R 172 H}$ allele dramatically accelerates malignant progression of skin tumors compared with loss of $p 53$. Of note, carcinoma formation in K-ras-p53/WT mice was accelerated compared with K-ras-p53 ${ }^{\mathrm{WT} / \mathrm{WT}}$ littermates, in agreement with the accelerated malignant progression observed in homozygous and heterozygous p53 knockout mice subjected to 2-stage chemical carcinogenesis protocols (27), thereby confirming that loss of p53 plays a role in skin cancer progression.

Since mutant p53 can bind and inactivate WT p53 (28), our results could be interpreted either as a consequence of the acquisition of gain-of-function properties of mutant $\mathrm{p} 53^{\mathrm{R} 172 \mathrm{H}}$ or due to dominant-negative effects of $\mathrm{p} 53^{\mathrm{R} 172 \mathrm{H}}$ toward WT $\mathrm{p} 53$. To address this question we generated mice that, in addition to carrying the $K-$ ras $^{G 12 D}$ allele, also carry either $1 p 53^{R 172 H}$ allele and $1 p 53^{f}$

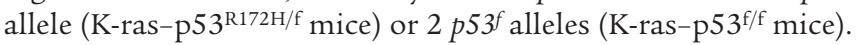
After activation of Cre in the skin by treatment with RU486, the 


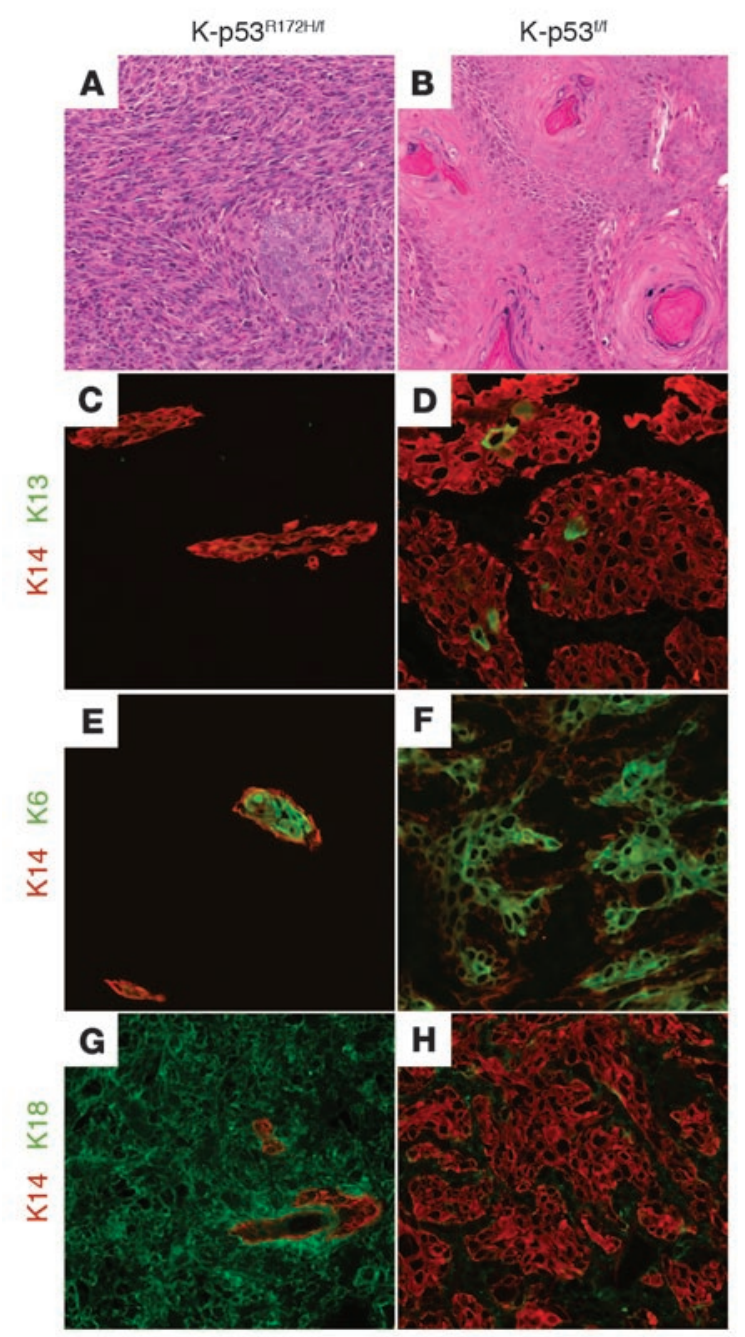

K-ras-p53 $3^{\mathrm{R} 172 \mathrm{H} / \mathrm{f}}$ mice express mutant $\mathrm{p} 53^{\mathrm{R} 172 \mathrm{H}}$ in the absence of WT p53. Therefore, a dominant-negative effect for $\mathrm{p} 53^{\mathrm{R} 172 \mathrm{H}}$ is ruled out in this model. Tumor formation in K-ras-p53 $172 \mathrm{H} / \mathrm{f}$ mice was compared with K-ras-p53/f mice, which do not express p53 in Cre-activated cells. We observed that after treatment with TPA, the $\mathrm{K}$-ras-p53 $3^{\mathrm{R} 172 \mathrm{H} / \mathrm{f}}$ mice developed more tumors than K-ras-p53/f mice (Figure 2E), and conversion to malignant carcinomas was also significantly accelerated in K-ras-p53 $3^{\mathrm{R} 172 \mathrm{H} / \mathrm{f}}$ mice compared with K-ras-p53 ${ }^{\mathrm{f} / \mathrm{f}}$ mice (Figure $2 \mathrm{~F}$ ). Activation of the conditional alleles in the skin tumors that developed in these mice was confirmed by PCR using specific primers for the activated alleles (Figure $2 \mathrm{G}$ ). These findings were supported by the detection of mutant p53 in the nuclei of skin tumors that developed in K-ras-p53 $172 \mathrm{H} / \mathrm{f}$ mice, primarily in the basal cells of the epithelial component of the tumors (Supplemental Figure 1; supplemental material available online with this article; doi:10.1172/JCI31721DS1), confirming that these tumors originated from epithelial cells that had activated the mutant $p 53^{R 172 H}$ allele.

Expression of endogenous $p 53^{R 172 H}$ results in formation of malignant skin carcinomas and metastasis. Histological analysis revealed that $60 \%$ of the carcinomas that developed in K-ras-p53 ${ }^{\mathrm{R} 172 \mathrm{H}}$ mice were spindle cell carcinomas that consisted primarily of epithelial cells that showed little differentiation and large areas of spindle cells, which

\section{Figure 3}

K-ras-p53 ${ }^{\mathrm{R} 172 \mathrm{H}}$ mice developed spindle cell carcinomas. (A and B) Hematoxylin and eosin staining of skin carcinomas that developed in K-ras-p53R172H/f mice (A) and K-ras-p53/// mice (B). (C-H) Keratin staining in carcinomas: double immunofluorescence for K14 (red) and K13 (green) (C and D), K14 (red) and K6 (green) (E and F), and K14 (red) and $\mathrm{K} 18$ (green) ( $\mathbf{G}$ and $\mathbf{H}$ ) on frozen sections obtained from carcinomas that developed in K-ras-p53 $3^{\mathrm{R} 172 \mathrm{H} / \mathrm{s}}$ mice $(\mathbf{C}, \mathbf{E}$, and $\mathbf{G}$ ) or $\mathrm{K}$-ras-p53/// mice (D, F, and $\mathbf{H})$. Original magnification, $\times 100$.

are the most undifferentiated and malignant cells in this type of tumor (29) (Figure 3A). In contrast, carcinomas that arose in $\mathrm{K}$-ras-p53 $\mathrm{f} / \mathrm{f}$ mice were differentiated SCCs with abundant keratin pearls and an absence of spindle cells (Figure 3B).

A more detailed characterization of tumor differentiation using antibodies specific for keratins revealed that K14 was only expressed in restricted areas of the K-ras-p $53^{\mathrm{R} 172 \mathrm{H} / \mathrm{f}}$ carcinomas (Figure 3C). These K14-positive areas of the tumors failed to express K13, a molecular marker for early stages of skin carcinogenesis (30) (Figure 3C). In addition, these tumors induced expression of the simple epithelial K18, a keratin that is not expressed in normal epidermis but that has been associated with late stages of skin carcinogenesis (31). K18 was expressed in epithelial areas of the tumors that were negative for K14 (Figure 3G). These observations are in contrast with the widespread expression of K14 in the K-ras-p53/f/ carcinomas, which also expressed $\mathrm{K} 13$ in the most differentiated areas of the tumor (Figure 3D). Moreover, the $\mathrm{K}$-ras-p53 ${ }^{\mathrm{f} / \mathrm{f}}$ carcinomas showed complete absence of K18 (Figure $3 \mathrm{H}$ ), again suggesting that these tumors represent earlier stages of progression compared with the K-ras-p53 $3^{\mathrm{R} 172 \mathrm{H} / \mathrm{f}}$ carcinomas. The proliferation-associated K6 was expressed in both K-ras-p53 ${ }^{\mathrm{R} 172 \mathrm{H} / \mathrm{f}}$ (Figure $3 \mathrm{E}$ ) and K-ras-p53 $3^{\mathrm{f} / \mathrm{f}}$ carcinomas (Figure 3F). Overall, this analysis demonstrates that carcinomas that arise as a result of activation of an endogenous $p 53^{R 172 H}$ gain-of-function mutation express keratin markers associated with malignant progression and present a more undifferentiated aggressive phenotype compared with tumors that arise as a result of loss of p53.

In support of these findings, we found that over $60 \%$ of the K-ras-p53 ${ }^{\mathrm{R} 172 \mathrm{H}}$ mice developed metastasis in lungs and/or lymph nodes (Table 1). This is a remarkable observation, as metastases are seldom observed in WT mice subjected to skin carcinogenesis protocols and were also absent in K-ras-p53 $3^{\mathrm{f} / \mathrm{f}}$ mice, which indicates that endogenous $p 53^{R 172 H}$ promotes the metastatic potential

\section{Table 1}

Mice that express p53 ${ }^{\mathrm{R} 172 \mathrm{H}}$ develop metastases in the lungs and/or lymph nodes

\section{Mice with metastasis/total mice analyzed (\%)}

\begin{tabular}{|c|c|c|}
\hline Genotype & $\begin{array}{l}\text { Analyzed by } \\
\text { genotype }\end{array}$ & $\begin{array}{c}\text { Analyzed by } \\
\text { p53 } \\
\text { R172H } \text { expression }^{A}\end{array}$ \\
\hline K-ras-p53 & $8 / 11(72 \%)$ & $13 / 21(62 \%)$ \\
\hline K-ras-p53 R172H/wT & $5 / 10(50 \%)$ & \\
\hline K-ras-p53//f & $0 / 9(0 \%)$ & \\
\hline K-ras-p53/WT & $1 / 4(25 \%)$ & $1 / 20(5 \%)$ \\
\hline K-ras-p53WT/wT & $0 / 7(0 \%)$ & \\
\hline
\end{tabular}

AData compiled for all mice that carry the $p 53^{R 172 H}$ allele and for all mice that do not carry the $p 53^{R 172 H}$ allele. 

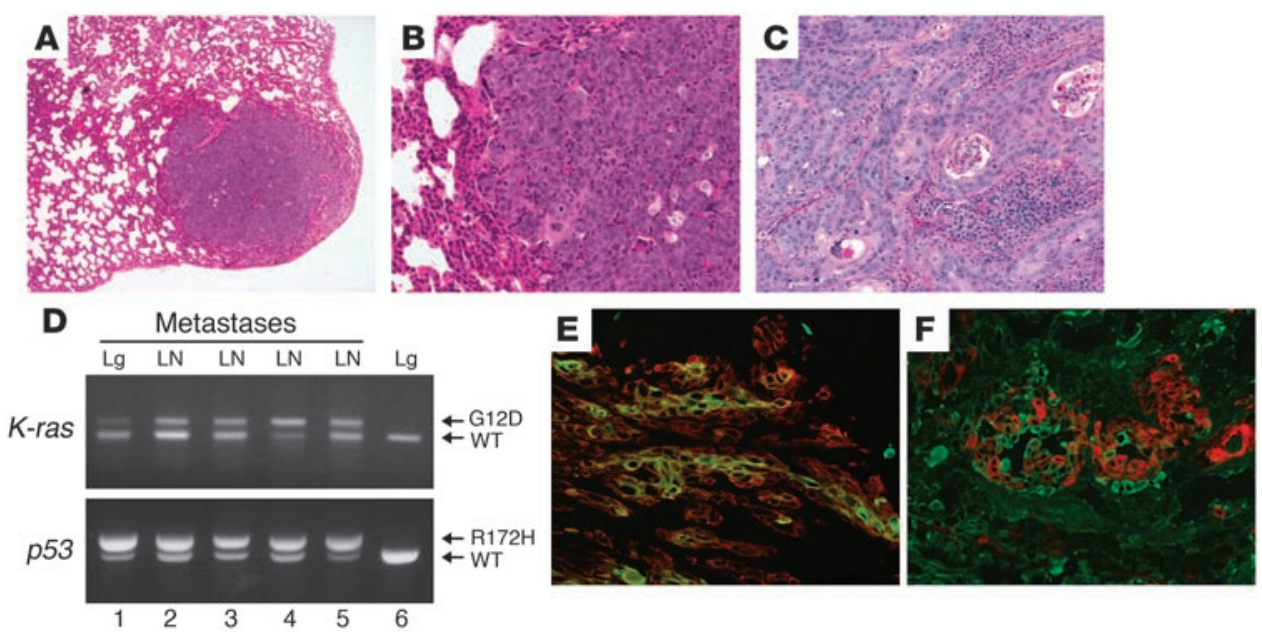

Figure 4

K-ras-p53 $172 \mathrm{H}$ tumors metastasize to lungs and lymph nodes. (A) Hematoxylin and eosin staining of a lung metastasis that developed in a K-ras-p53 $3^{\mathrm{R} 172 \mathrm{H} / \mathrm{f}}$ mouse. (B) Higher magnification of the metastasis shown in A. (C) Hematoxylin and eosin staining of a lymph node metastasis that developed in a K-rasp53 ${ }^{\mathrm{R} 172 \mathrm{H} / \mathrm{f}}$ mouse. Note the massive presence of metastatic epithelial cells invading the organs. (D) Activation of the $K$-ras ${ }^{G 12 D}$ and $p 53^{R 172 H}$ alleles in lung $(\mathrm{Lg})$ and lymph node $(\mathrm{LN})$ metastases (lanes $\left.1-5\right)$ using primers described in Figure 1B and Figure 2A. PCR analysis of DNA purified from lungs (lane 6) of a K-ras-p53 $172 \mathrm{H} / \mathrm{WT}$ mouse was used as control. (E and F) Double immunofluorescence for K14 (red) and K6 (green) (E) and K18 (green) and K14 (red) (F) on frozen sections obtained from lymph node metastases that developed in K-ras-p53 $172 \mathrm{H} / \mathrm{f}$ mice. Original magnification, $\times 40(\mathbf{A}), \times 100(\mathbf{B}-\mathbf{C}, \mathbf{E}$, and F).

cover more than $98 \%$ of the mouse genome. We found that $42 \%(8 / 19)$ of the K-ras-p53 $3^{\mathrm{R} 172 \mathrm{H}}$ carcinomas analyzed exhibited chromosome gains, compared with $23 \%(3 / 13)$ of the K-ras-p53 ${ }^{\mathrm{f}}$ and $20 \%(1 / 5)$ of the K-ras-p53 ${ }^{\mathrm{WT}}$ carcinomas, indicating that aneuploidy is more frequent in carcinomas that express the gain-of-function $\mathrm{p} 53^{\mathrm{R} 172 \mathrm{H}}$ than in tumors that lose p53 or that express WT p53. Interestingly, gains of chromosomes 15 (3/19) and $3(3 / 19)$ were only observed in K-ras-p53 ${ }^{\mathrm{R} 172 \mathrm{H}}$ carcinomas (Figure $5, \mathrm{D}$ and $\mathrm{E}$ ), whereas gain of chromosome 6 was observed in tumors of all genotypes.

Because over $50 \%$ of the K-ras$\mathrm{p} 53^{\mathrm{R} 172 \mathrm{H}}$ carcinomas analyzed did not show chromosome amplification in BAC-CGH arrays, we analyzed ploidy on metaphase spreads obtained from tumor cells cultured from 6 K-ras-p53 $3^{\mathrm{R} 172 \mathrm{H}}$ carcinomas. We found that most of the tumor cells contained 70-90 chromosomes, a near-tetraploid

of skin cancers. Histological examination demonstrated that the lungs and lymph nodes of mice with metastasis had been invaded by epithelial tumor cells (Figure 4, A-C). In addition, we confirmed that these metastases contained activated $K$-ras ${ }^{G 12 D}$ and $p 53^{R 172 H}$ alleles (Figure 4D). The presence of epithelial tumor cells in the metastases was further confirmed by staining with keratin antibodies, which revealed focal areas of cells that expressed K14, K6, and K18 (Figure 4, E and F). Although spindle cells, which originate by epithelial-to-mesenchymal transition, are considered to be the most malignant cells in skin cancers, our results suggest that cells that maintain expression of epithelial markers can also metastasize, as previously demonstrated in other mouse models (32). Overall, these results confirmed that the metastases originated from epithelial tumor cells that had activated the $K$-ras $^{G 12 D}$ and $p 53^{R 172 H}$ alleles and documented that the $p 53^{R 172 H}$ allele confers gain-of-function properties that accelerate malignant progression and metastasis of skin cancers.

The $p 53^{R 172 H}$ tumors exhibit centrosome amplification and aneuploidy. Previous mouse models have shown that skin and mammary tumors that overexpress $\mathrm{p} 53^{\mathrm{R} 172 \mathrm{H}}$ exhibit centrosome amplification $(20,33)$, a process that may result in genomic instability (34). To determine whether centrosome amplification also occurred in skin tumors that express the $p 53^{R 172 H}$ allele, we stained frozen sections of skin papillomas with an antibody specific for centrosomes (35). This analysis detected supernumerary centrosomes in 3\%-5\% of the cells in K-ras-p53 $3^{\mathrm{R} 172 \mathrm{H} / \mathrm{f}}$ tumors (Figure 5, A and B), but not in tumors that developed in K-ras-p53 ${ }^{\mathrm{f} / \mathrm{f}}$ mice (Figure $5 \mathrm{C}$ ). Since centrosome amplification may promote tumor progression through mechanisms involving aneuploidy (34), we analyzed the genomic content of skin carcinomas that developed in these mice using comparative genomic hybridization on bacterial artificial chromosome (BAC-CGH) containing over 35,000 BAC clones, which
DNA content, which is difficult to detect by BAC-CGH arrays (Figure 5F). FISH analysis of the metaphase spreads using whole chromosome painting probes confirmed the presence of 4 copies of chromosome 2 (Figure $5 \mathrm{G}$ ), a chromosome not found amplified in the BAC-CGH assays. In addition, multiple copies of chromosome 6 were detected in cells derived from tumors that showed chromosome 6 amplification by BAC-CGH (Figure 5H). These findings indicate that skin carcinomas that developed in these models displayed a near-tetraploid DNA content, with increased chromosome gains in tumors that expressed endogenous $p 53^{R 172 H}$. Overall, these results suggest that expression of the gain-of-function $p 53^{R 172 H}$ allele in skin tumors predisposes to centrosome amplification, which may result in accelerated aneuploidy and malignant progression of skin carcinomas.

\section{Discussion}

Extensive evidence obtained from clinical studies suggests that mutations in ras and $p 53$ genes are involved in human NMSC development. In addition, studies based on transgenic mouse models and chemical carcinogenesis experiments support a causal role for ras mutations in skin cancer initiation, whereas mutations in $p 53$ may have a prominent role in later stages of malignant progression (36). Although these observations suggest that ras and p53 mutations may cooperate during skin carcinogenesis, prior to this study, in vivo models to assess cooperation between endogenous ras and $p 53$ mutations had not, to our knowledge, been reported. Since most $p 53$ mutations found in human cancers are gain-of-function rather than loss-of-function mutations, comparing the effects of gain-of-function and loss-of-function mutations was critical to understanding their contrasting roles during skin carcinogenesis. The inducible mouse model described here allowed us to demonstrate that endogenous ras and gain-of-func- 

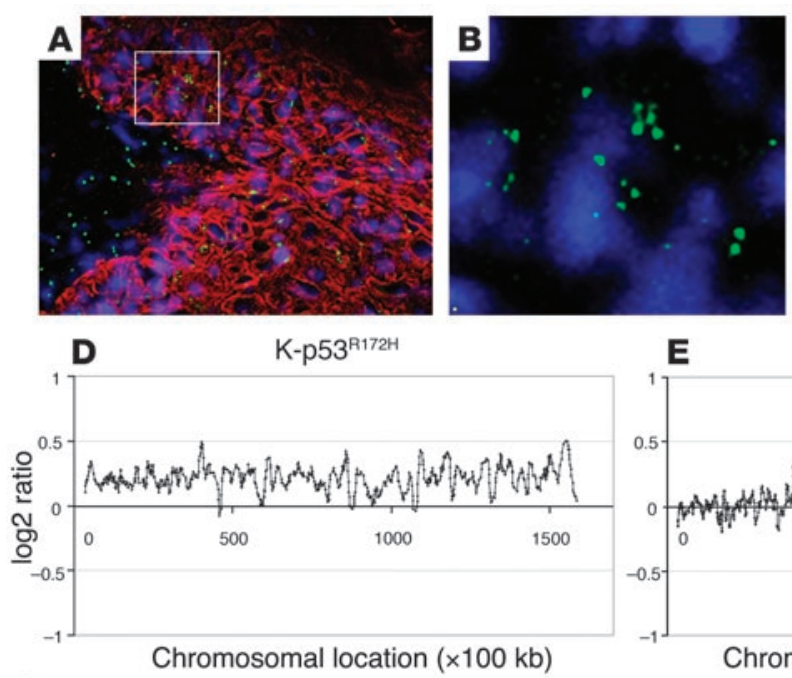

E
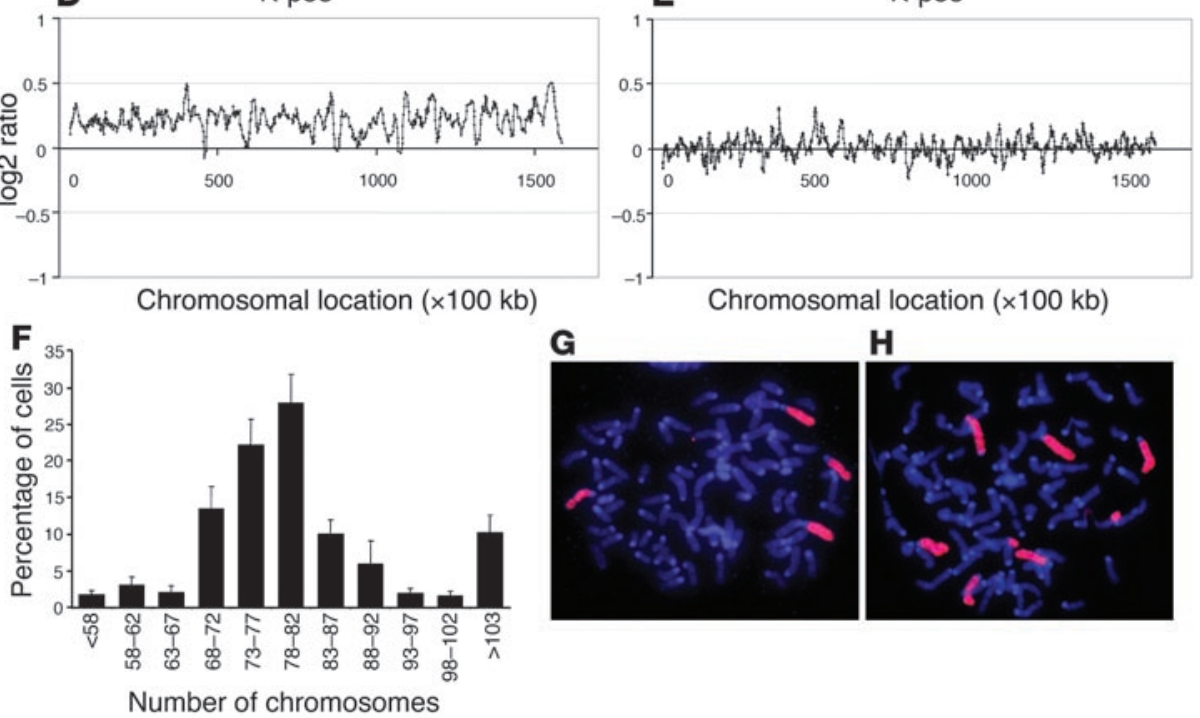

G

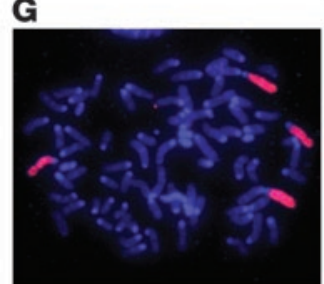

H

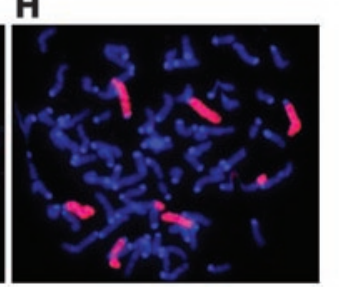

\section{Figure 5}

K-ras-p53R172H tumors exhibit centrosome amplification and aneuploidy. (A-C) Double immunofluorescence for centrosomes (green) and K14 (red) in papillomas that developed in

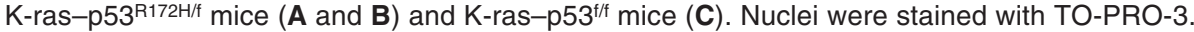
Note the presence of cells with more than 2 centrosomes in K-ras-p53R172H/f tumors (A) but not in $\mathrm{K}$-ras-p53/f/ tumors (C). (B) Higher magnification of the area within the white square shown in A. The red staining for $\mathrm{K} 14$ was omitted to allow better visualization of multiple centrosomes in the cells. (D and E) BAC-CGH genomic profile for chromosome 3 in a K-ras-p53R172H carcinoma (D) and a K-ras-p53/f/ carcinoma (E) obtained by plotting log2 ratios for each BAC clone ( $y$ axis) according to their chromosomal location ( $x$ axis). Note gains throughout the entire chromosome

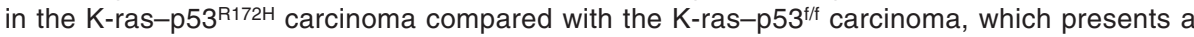
mostly stable profile. (F) Chromosome counts in metaphase spreads obtained from 6 independent K-ras-p53 ${ }^{\mathrm{R} 172 \mathrm{H}}$ carcinomas. (G and $\mathbf{H}$ ) FISH for chromosome 2 (G) and chromosome 6 (H) using whole-chromosome painting probes on metaphase spreads from K-ras-p53 ${ }^{\mathrm{R} 172 \mathrm{H}}$ carcinomas. Magnification, $\times 100(\mathbf{A}$ and $\mathbf{C}), \times 1000(\mathbf{G}$ and $\mathbf{H}), \times 2000(\mathbf{B})$.

tion $p 53$ mutations cooperate in skin cancer initiation, malignant progression, and metastasis.

Previously, several groups including our own have shown that overexpression of oncogenic ras in the skin can result in tumor formation (9-12). Since mutant ras was overexpressed in these mice, these models did not allow assessment of whether endogenous ras mutations, which occur in sporadic human cancers, are sufficient to initiate skin cancer formation. Skin papilloma formation was also reported in a subset of mice that expressed the $K$-ras ${ }^{G 12 D}$ allele in the skin (37). However, the reason for the incomplete penetrance of the tumor phenotype in these mice was unclear. In this regard, we found that skin tumor formation in the $\mathrm{K} 5 \mathrm{Cr} \mathrm{C}^{*}$-Ras mice only occurs after treatment with the tumor promoter TPA or in areas of the skin subjected to chronic injury, such as ear tags. Similar findings were also reported for mice that overexpressed oncogenic $\mathrm{H}$-ras in the skin under the control of the $\mathrm{K} 1$ or $\mathrm{K} 10$ promoter opment. This analysis demonstrated that activation of a mutant $p 53^{R 172 H}$ allele, but not loss of $p 53$, cooperates with $K$-ras ${ }^{G 12 D}$ in skin cancer initiation. Interestingly, patches of cells that contain missense mutations in $p 53$ are also found in sun-exposed normal human skin $(42,43)$. However, skin tumors very seldom arise from these mutant $p 53$ cells, suggesting that additional mutations may be required for tumor development. Our observations indicate that the presence of $p 53$ gain-of-function mutations in epidermal cells can facilitate skin tumor formation when additional genetic alterations such as ras mutations accumulate in these cells. Since tumors that have mutations in $p 53$ do not always carry mutations in ras genes, it is tempting to speculate that $p 53$ mutations may also cooperate with other genetic alterations to initiate skin cancer.

Mice subjected to chemical carcinogenesis protocols exhibit $p 53$ mutations in SCCs, but not in benign papillomas, suggesting a role for $p 53$ mutations in skin cancer progression $(44,45)$. Accordingly, 
p53-null mice treated with DMBA/TPA exhibit accelerated tumor progression, indicating that loss of $p 53$ predisposes to malignant conversion of skin tumors $(27,46)$. In agreement with these findings, we also observed increased carcinoma formation after deletion of the $p 53$ gene in the presence of the K-ras ${ }^{G 12 D}$ allele. More striking, however, was our finding that mice that expressed the gain-of-function $p 53^{R 172 H}$ mutation exhibited accelerated malignant progression and a much more aggressive tumor phenotype, including the ability to develop metastasis, compared with mice that exhibited loss of $p 53$. These observations clearly indicate that the endogenous $p 53^{R 172 H}$ mutation confers gain-of-function properties involved in malignant progression and metastasis of skin cancers and represent the strongest in vivo evidence to date for an oncogenic role for endogenous $p 53$ missense mutations. Our findings are in agreement with previous reports showing that mice carrying knockin $p 53^{R 172 H}$ or $p 53^{R 270 H}$ alleles in the germline developed tumor spectra different from $p 53$-null mice, with increased metastatic potential $(18,19)$. Unfortunately, an assessment of the role of gain-of-function p53 mutants in skin carcinogenesis was not possible in these initial reports, since mice homozygous for these mutant $p 53$ alleles succumbed to a wide variety of internal tumors within 5 to 6 months. In the present study, we overcame this limitation of the constitutive knockin model by activating the conditional $p 53^{R 172 H}$ allele in somatic epidermal cells. The use of this approach in other tissues may reveal the role of $p 53$ gain-offunction mutations in other tumor types. In addition, since the oncogenic roles of different p53 mutations may differ (47), similar studies will be required to determine the impact of other potential p53 gain-of-function mutations in tumorigenesis.

The presence of supernumerary centrosomes in K-ras-p53 $3^{\mathrm{R} 172 \mathrm{H} / \mathrm{f}}$ mice but not in K-ras-p53/f mice may provide a mechanistic explanation for the oncogenic role of $p 53^{R 172 H}$ during skin carcinogenesis. Abnormal centrosome amplification can lead to defective chromosome segregation, generally resulting in chromosome contents incompatible with cell viability. However, a small proportion of cells may acquire a growth advantage as a result of the gain of chromosomes that carry oncogenes or loss of chromosomes that carry tumor suppressor genes. In fact, we found a correlation between centrosome amplification and accelerated aneuploidy, which may contribute to the malignant properties of the tumors that developed in the K-ras-p53 $3^{\mathrm{R} 172 \mathrm{H}}$ mice. Similarly, genomic instability was also found in pancreatic tumors that developed in mice that express $K-$ ras $^{G 12 D}$ and $p 53^{R 172 H}$ (48), although it was unclear in that model whether genomic instability was the result of gain-of-function or loss of p53 activity. Despite substantial evidence to suggest that centrosome amplification may lead to genomic instability, we cannot exclude the possibility that centrosome abnormalities arise in tumors that express mutant p53 as a consequence of the more aggressive phenotype of these tumors.

There is a very interesting correlation between the results obtained with our mouse models and the types of p53 mutations found in human skin cancers. Notably, most of the p53 mutations found in human SCCs are point mutations that often coexist in the presence of the WT p53 allele, a mutation pattern expected for gain-of-function mutations (14). However, the more benign basal cell carcinomas primarily exhibit mutations that introduce stop codons, and both p53 alleles are frequently mutated or deleted (49). Furthermore, SCCs tend to be aneuploid, whereas basal cell carcinomas are largely diploid. Thus, our observations may provide a causal relation between p53 gain-of-function mutations and the overall more aggressive tumor phenotype found in human skin cancers that harbor this type of mutation.

Studies performed on osteosarcoma cells derived from the $p 53^{R 172 H}$ germline mutant mice suggested that mutant p53 in these cells may exert its effects, at least in part, through binding and inactivation of the p53 family members p63 and p73 $(18,19)$. However, we did not detect binding of $\mathrm{p} 53^{\mathrm{R} 172 \mathrm{H}}$ to $\mathrm{p} 63$ or $\mathrm{p} 73$ in epidermal keratinocytes, nor did we find modulation of the p63 and $\mathrm{p} 73$ transactivation properties by $\mathrm{p} 53^{\mathrm{R} 172 \mathrm{H}}$ (Supplemental Figure 2). These results are not surprising considering that the predominant $\mathrm{p} 63$ and $\mathrm{p} 73$ isoforms expressed in the skin are the dominant-negative $\Delta \mathrm{N}$ isoforms and considering the recent finding that TAp63 has tumor-promoting properties in the skin rather than a tumor suppressor role (50). In addition, Flores et al. have shown that mice heterozygous for either p53 and p63 or p53 and p73 develop broader tumor spectrum than $\mathrm{p} 53^{+/-}$mice. However, skin tumors were not reported in this study (51), further suggesting that inactivation of $\mathrm{p} 63$ and $\mathrm{p} 73$ does not play a role in skin cancer development. This scenario suggests that mutant p53 could use different mechanisms to induce malignant properties in epidermal keratinocytes. In this regard, a recent report proposed that mutant p53 can bind and inactivate the NF-Y transcription factor, thereby expanding the spectrum of molecular mechanisms that may contribute to the oncogenic properties of mutant p53 (52). Although the role of the NF-Y complex in skin cancer is presently unknown, these findings suggest that mutant p53 may exert its oncogenic effects through a variety of molecular mechanisms that may function in a tissue-specific manner.

In summary, our study provides in vivo evidence documenting cooperation between endogenous ras and p53 mutations during skin carcinogenesis and clearly demonstrates an oncogenic role for the gain-of-function mutation $p 53^{R 172 H}$ in skin cancer development. These observations have profound implications for the future design of antitumor therapies for tumors that carry putative gain-of-function mutations in $p 53$, as these therapies should not only restore WT p53 function but also inactivate the gain-offunction properties of mutant p53.

\section{Methods}

Mouse models. Generation of K5.Cre*PR1 and Neo-p53 ${ }^{\mathrm{R} 172 \mathrm{H}}$ (previously named Neo-p53 $\left.{ }^{515 A}\right)$ mice has been described $(18,21)$. LSL-K-ras ${ }^{\mathrm{G} 12 \mathrm{D}}$ mice were provided by Tyler Jacks (Massachusetts Institute of Technology Center for Cancer Research, Cambridge, Massachusetts, USA) and David Tuveson (University of Pennsylvania School of Medicine, Philadelphia, Pennsylvania, USA) (22). Floxed p53 mice were provided by Anton Berns (Netherlands Cancer Institute, Amsterdam, The Netherlands) (26). The genetic backgrounds of the mouse lines were as follows: K5.Cre*PR1, C57BL/6; Neo-p53 ${ }^{\mathrm{R} 172 \mathrm{H}}, 129 \mathrm{~Sv} / \mathrm{C} 57 \mathrm{BL} / 6$; LSL-K-ras ${ }^{\mathrm{G} 12 \mathrm{D}}, 129 \mathrm{~Sv} / \mathrm{C} 57 \mathrm{BL} / 6$; floxed p53, FVB/129Sv. K5.Cre* PR1 mice were crossed to LSL-K-ras ${ }^{\mathrm{G} 12 \mathrm{D}}$ mice to generate K5.Cre*PR1/LSL-K-ras ${ }^{\mathrm{G} 12 \mathrm{D}}$ (K5Cre*-Ras). Neo-p53 ${ }^{\mathrm{R} 172 \mathrm{H}}$ mice were crossed with floxed p53 to generate Neo-p53 $3^{\mathrm{R} 172 \mathrm{H} / \mathrm{f}}$ mice. $\mathrm{K} 5 \mathrm{Cre}{ }^{*}$-Ras mice were crossed with Neo-p53 $3^{\mathrm{R} 172 \mathrm{H} / \mathrm{f}}$ to generate $\mathrm{K} 5 . \mathrm{Cre}{ }^{*} \mathrm{PR} 1 / \mathrm{LSL}-\mathrm{K}-\mathrm{ras}^{\mathrm{G} 12 \mathrm{D}} /$ Neo-p53 $3^{\mathrm{R} 172 \mathrm{H} / \mathrm{WT}}\left(\mathrm{K}\right.$-ras-p53 $\left.3^{\mathrm{R} 172 \mathrm{H} / \mathrm{WT}}\right)$ and K5.Cre*PR1/LSL-K-ras ${ }^{\mathrm{G} 12 \mathrm{D} /}$ $\mathrm{p} 53^{\mathrm{f} / \mathrm{WT}}\left(\mathrm{K}-\mathrm{ras}-\mathrm{p} 53^{\mathrm{f} / \mathrm{WT}}\right)$ mice in a mixed genetic background (FVB/129Sv/ C57BL/6). Additional matings between Neo-p53 $172 \mathrm{H} / \mathrm{f}$ and K-ras-p53/WT mice were established to generate K-ras- $\mathrm{p} 53^{\mathrm{R} 172 \mathrm{H} / \mathrm{f}}$ and K-ras- $\mathrm{p} 53^{\mathrm{f} / \mathrm{f}}$ mice. Excision of the stop cassette from the Neo-p53 ${ }^{\mathrm{R} 172 \mathrm{H}}$ allele was analyzed using the following primers: R172H reverse, GGAGCCAGGCCTAAGAGCAAGAATAAGTCA, and R172H forward, GACCCCTTCTCACCAAAAACAAAAACAGC. Analysis of excision of the stop cassette from the 
LSL-K-ras ${ }^{\mathrm{G} 12 \mathrm{D}}$ allele and excision of the loxP-flanked sequences from the floxed p53 allele was performed as described in refs. 22 and 26. All experiments involving mice were approved by the Institutional Animal Care and Use Committee of Baylor College of Medicine.

Histological analysis and immunofluorescence. For histology, biopsied tumors were fixed overnight in $10 \%$ neutral-buffered formalin at room temperature and then transferred to $75 \%$ ethanol, embedded in paraffin, sectioned, and stained with hematoxylin and eosin. For immunofluorescence, tumor biopsies were embedded in OCT compound and frozen at $-70^{\circ} \mathrm{C}$, and $5-\mu \mathrm{m}$ sections were obtained and subjected to double-label immunofluorescence using the p53 antibody CM5p (Novacastra) and polyclonal antibodies for K14, K13, and K6 (53). OCT sections were fixed in methanol for 10 minutes for $\mathrm{p} 53$ detection and in $75 \%$ acetone $/ 25 \%$ ethanol for 15 minutes for keratins, then blocked in 5\% BSA and incubated overnight at room temperature with the primary antibody mixes indicated in Figure 1, C and F, Figure 3, $\mathrm{C}-\mathrm{H}$, and Figure 4, E and F. The sections were then washed with PBS and incubated with secondary antibodies conjugated to Alexa Fluor 488 or 594 dyes (Invitrogen) for 45 minutes at room temperature. Images from stained sections were acquired using a Nikon Eclipse E600 microscope and MetaVue Meta Imaging Series 6.2 software (Molecular Devices) and processed with Adobe Photoshop 6.0.

Confocal imaging and scoring of centrosomes. OCT-embedded tumors were sectioned at $50 \mu \mathrm{m}$ using a minitome cryostat. The thick frozen sections were mounted onto positively charged slides, fixed, and stained for centrosomes as described previously (35). Sections were costained with the antiK14 antibody and TO-PRO-3 iodide (Invitrogen). Immunofluorescence microscopy was performed with a confocal microscope (Zeiss LSM 510). Scoring was accomplished by counting the number of stained nuclei and the corresponding number of centrosomes in tissue sections (35). A minimum of 300 nuclei/sample were counted.

BAC-CGH. Genome-wide analysis of skin carcinomas was performed as previously described (54) using BAC microarrays containing 35,000 BAC clones.
Metaphase spreads and FISH. Tumor cells growing in $100-\mathrm{mm}$ plates were incubated with $2.5 \mu \mathrm{g} / \mathrm{ml}$ colchicine for 2 hours. After washing with PBS, the cells were detached with trypsin, resuspended in $5 \mathrm{ml}$ medium, and centrifuged at $200 \mathrm{~g}$ for 5 minutes. The pellet was washed with PBS, resuspended in $0.056 \mathrm{M}$ $\mathrm{KCl}$, and left at room temperature for 30 minutes. After spinning at $200 \mathrm{~g}$ for 5 minutes, the pellet was resuspended in $75 \%$ methanol $/ 25 \%$ acetic acid, a process that was repeated 2 more times. The cell suspension was spread in drops onto a cold and wet slide, allowed to dry at room temperature, and mounted using VECTASHIELD Mounting Media with DAPI (Vector Laboratories). For FISH analysis, metaphase spreads prepared as described above were hybridized with biotinylated whole-chromosome painting probes (Open Biosystems) for mouse chromosomes 6 and 2 according to the manufacturer's instructions. Chromosomes were visualized by incubation with Texas Red Streptavidin (Vector Laboratories). Images were acquired using a Nikon Eclipse E600 microscope and MetaVue Meta Imaging Series 6.2 software (Molecular Devices).

\section{Acknowledgments}

C. Caulin was supported by a Research Career Development Award from the Dermatology Foundation. This work was supported by NIH grants CA52607 to D.R. Roop, DE015344 to C. Caulin, CA105491 to D.R. Roop and C. Caulin, CA41424 to B.R. Brinkley, and CA64255 to B.R. Brinkley and T.M. Goepfert.

Received for publication February 6, 2007, and accepted in revised form April 24, 2007.

Address correspondence to: Dennis R. Roop, UCDHSC at Fitzsimons, Mail Stop 8320, RM P18-8129, P.O. Box 6511, Aurora, Colorado 80045, USA. E-mail: Dennis.Roop@UCHSC.edu. Or to: Carlos Caulin, MD Anderson Cancer Center, Department of Head and Neck Surgery, Unit 123, 1515 Holcombe Blvd., RM T5.3895, Houston, Texas 77030, USA. Phone: (713) 794-5603; Fax: (713) 745-2234; E-mail: ccaulin@mdanderson.org.
1. Alam, M., and Ratner, D. 2001. Cutaneous squamous-cell carcinoma. N. Engl. J. Med. 344:975-983.

2. Albert, M.R., and Weinstock, M.A. 2003. Keratinocyte carcinoma. CA Cancer J. Clin. 53:292-302.

3. Boukamp, P. 2005. Non-melanoma skin cancer: what drives tumor development and progression? Carcinogenesis. 26:1657-1667.

4. Pierceall, W.E., Goldberg, L.H., Tainsky, M.A., Mukhopadhyay, T., and Ananthaswamy, H.N. 1991. Ras gene mutation and amplification in human nonmelanoma skin cancers. Mol. Carcinog. 4:196-202.

5. Spencer, J.M., Kahn, S.M., Jiang, W., DeLeo, V.A., and Weinstein, I.B. 1995. Activated ras genes occur in human actinic keratoses, premalignant precursors to squamous cell carcinomas. Arch. Dermatol. 131:796-800

6. Balmain, A., and Brown, K. 1988. Oncogene activation in chemical carcinogenesis. Adv. Cancer Res. 51:147-182.

7. Ise, K., et al. 2000. Targeted deletion of the H-ras gene decreases tumor formation in mouse skin carcinogenesis. Oncogene. 19:2951-2956.

8. Rehman, I., et al. 2000. Frequent codon 12 Kiras mutations in mouse skin tumors initiated by $\mathrm{N}$-methyl-N'-nitro-N-nitrosoguanidine and promoted by mezerein. Mol. Carcinog. 27:298-307.

9. Bailleul, B., et al. 1990. Skin hyperkeratosis and papilloma formation in transgenic mice expressing a ras oncogene from a suprabasal keratin promoter. Cell. 62:697-708.

10. Brown, K., Strathdee, D., Bryson, S., Lambie, W., and Balmain, A. 1998. The malignant capacity of skin tumours induced by expression of a mutant $\mathrm{H}$-ras transgene depends on the cell type targeted.
Curr. Biol. 8:516-524.

11. Greenhalgh, D.A., et al. 1993. Induction of epidermal hyperplasia, hyperkeratosis, and papillomas in transgenic mice by a targeted v-Ha-ras oncogene. Mol. Carcinog. 7:99-110.

12. Vitale-Cross, L., Amornphimoltham, P., Fisher, G., Molinolo, A.A., and Gutkind, J.S. 2004. Conditional expression of K-ras in an epithelial compartment that includes the stem cells is sufficient to promote squamous cell carcinogenesis. Cancer Res. 64:8804-8807.

13. Bolshakov, S., et al. 2003. p53 mutations in human aggressive and nonaggressive basal and squamous cell carcinomas. Clin. Cancer Res. 9:228-234.

14. Brash, D.E., et al. 1991. A role for sunlight in skin cancer: UV-induced p53 mutations in squamous cell carcinoma. Proc. Natl. Acad. Sci. U. S. A. 88:10124-10128.

15. Dittmer, D., et al. 1993. Gain of function mutations in p53. Nat. Genet. 4:42-46.

16. Sun, Y., Nakamura, K., Wendel, E., and Colburn, N. 1993. Progression toward tumor cell phenotype is enhanced by overexpression of a mutant p53 tumor-suppressor gene isolated from nasopharyngeal carcinoma. Proc. Natl. Acad. Sci. U. S. A. 90:2827-2831.

17. Cadwell, C., and Zambetti, G.P. 2001. The effects of wild-type p53 tumor suppressor activity and mutant p53 gain-of-function on cell growth. Gene. 277:15-30.

18. Lang, G.A., et al. 2004. Gain of function of a p53 hot spot mutation in a mouse model of Li-Fraumeni syndrome. Cell. 119:861-872.

19. Olive, K.P., et al. 2004. Mutant p53 gain of function in two mouse models of Li-Fraumeni syndrome. Cell. 119:847-860.

20. Wang, X.J., et al. 1998. Expression of a p53 mutant in the epidermis of transgenic mice accelerates chemical carcinogenesis. Oncogene. 17:35-45.

21. Caulin, C., et al. 2004. Inducible activation of oncogenic K-ras results in tumor formation in the oral cavity. Cancer Res. 64:5054-5058.

22. Jackson, E.L., et al. 2001. Analysis of lung tumor initiation and progression using conditional expression of oncogenic K-ras. Genes Dev. 15:3243-3248.

23. Roop, D.R., et al. 1989. Keratin expression in mouse epidermal tumors. Carcinog. Compr. Surv. 11:257-271.

24. Gimenez-Conti, I., et al. 1990. Early expression of type I K13 keratin in the progression of mouse skin papillomas. Carcinogenesis. 11:1995-1999.

25. Ko, L.J., and Prives, C. 1996. p53: puzzle and paradigm. Genes Dev. 10:1054-1072.

26. Jonkers, J., et al. 2001. Synergistic tumor suppressor activity of BRCA2 and p53 in a conditional mouse model for breast cancer. Nat. Genet. 29:418-425.

27. Kemp, C.J., Donehower, L.A., Bradley, A., and Balmain, A. 1993. Reduction of p53 gene dosage does not increase initiation or promotion but enhances malignant progression of chemically induced skin tumors. Cell. 74:813-822.

28. de Vries, A., et al. 2002. Targeted point mutations of p53 lead to dominant-negative inhibition of wild-type 53 function. Proc. Natl. Acad. Sci. U. S. A. 99:2948-2953.

29. Klein-Szanto, A.J. 1989. Pathology of human and experimental skin tumors. Carcinog. Compr. Surv. 11:19-53. 
30. Nischt, R., et al. 1988. Aberrant expression during two-stage mouse skin carcinogenesis of a type I 47-kDa keratin, K13, normally associated with terminal differentiation of internal stratified epithelia. Mol.Carcinog. 1:96-108.

31. Caulin, C., Bauluz, C., Gandarillas, A., Cano, A., and Quintanilla, M. 1993. Changes in keratin expression during malignant progression of transformed mouse epidermal keratinocytes. Exp. Cell Res. 204:11-21.

32. Han, G., et al. 2005. Distinct mechanisms of TGFbeta1-mediated epithelial-to-mesenchymal transition and metastasis during skin carcinogenesis. J. Clin. Invest. 115:1714-1723. doi:10.1172/JCI24399.

33. Murphy, K.L., Dennis, A.P., and Rosen, J.M. 2000. A gain of function p53 mutant promotes both genomic instability and cell survival in a novel p53-null mammary epithelial cell model. FASEB J. 14:2291-2302.

34. Brinkley, B.R. 2001. Managing the centrosome numbers game: from chaos to stability in cancer cell division. Trends Cell Biol. 11:18-21.

35. Goepfert, T.M., et al. 2002. Centrosome amplification and overexpression of aurora A are early events in rat mammary carcinogenesis. Cancer Res. 62:4115-4122.

36. Dlugosz, A., Merlino, G., and Yuspa, S.H. 2002. Progress in cutaneous cancer research. J. Investig. Dermatol. Symp. Proc. 7:17-26.

37. Tuveson, D.A., et al. 2004. Endogenous oncogenic K-ras(G12D) stimulates proliferation and widespread neoplastic and developmental defects. Can- cer Cell. 5:375-387.

38. Chan, I.T., et al. 2004. Conditional expression of oncogenic K-ras from its endogenous promoter induces a myeloproliferative disease. J. Clin. Invest. 113:528-538. doi:10.1172/JCI200420476.

39. Mao, J.H., et al. 2004. Mutually exclusive mutations of the Pten and ras pathways in skin tumor progression. Genes Dev. 18:1800-1805.

40. Owens, D.M., Spalding, J.W., Tennant, R.W., and Smart, R.C. 1995. Genetic alterations cooperate with v-Ha-ras to accelerate multistage carcinogenesis in TG.AC transgenic mouse skin. Cancer Res. 55:3171-3178.

41. Quintanilla, M., Brown, K., Ramsden, M., and Balmain, A. 1986. Carcinogen-specific mutation and amplification of Ha-ras during mouse skin carcinogenesis. Nature. 322:78-80.

42. Nakazawa, H., et al. 1994. UV and skin cancer: specific p53 gene mutation in normal skin as a biologically relevant exposure measurement. Proc. Natl. Acad. Sci. U. S. A. 91:360-364.

43. Jonason, A.S., et al. 1996. Frequent clones of p53mutated keratinocytes in normal human skin. Proc. Natl. Acad. Sci. U. S. A. 93:14025-14029.

44. Burns, P.A., et al. 1991. Loss of heterozygosity and mutational alterations of the p53 gene in skin tumours of interspecific hybrid mice. Oncogene. 6:2363-2369.

45. Ruggeri, B., et al. 1991. Alterations of the p53 tumor suppressor gene during mouse skin tumor progression. Cancer Res. 51:6615-6621.

46. Kelly-Spratt, K.S., Gurley, K.E., Yasui, Y., and Kemp,
C.J. 2004. p19Arf suppresses growth, progression, and metastasis of Hras-driven carcinomas through p53-dependent and -independent pathways. PLoS Biol. 2:E242.

47. Soussi, T., and Lozano, G. 2005. p53 mutation heterogeneity in cancer. Biochem. Biophys. Res. Commun. 331:834-842.

48. Hingorani, S.R., et al. 2005. Trp53R172H and KrasG12D cooperate to promote chromosomal instability and widely metastatic pancreatic ductal adenocarcinoma in mice. Cancer Cell. 7:469-483.

49. Ziegler, A., et al. 1993. Mutation hotspots due to sunlight in the p53 gene of nonmelanoma skin cancers. Proc. Natl. Acad. Sci. U. S. A. 90:4216-4220.

50. Koster, M.I., Lu, S.L., White, L.D., Wang, X.J., and Roop, D.R. 2006. Reactivation of developmentally expressed p63 isoforms predisposes to tumor development and progression. Cancer Res. 66:3981-3986.

51. Flores, E.R., et al. 2005. Tumor predisposition in mice mutant for $\mathrm{p} 63$ and $\mathrm{p} 73$ : evidence for broader tumor suppressor functions for the p53 family. Cancer Cell. 7:363-373.

52. Di Agostino, S., et al. 2006. Gain of function of mutant $\mathrm{p} 53$ : the mutant $\mathrm{p} 53 / \mathrm{NF}-\mathrm{Y}$ protein complex reveals an aberrant transcriptional mechanism of cell cycle regulation. Cancer Cell. 10:191-202.

53. Roop, D.R., et al. 1984. Synthetic peptides corresponding to keratin subunits elicit highly specific antibodies. J. Biol. Chem. 259:8037-8040.

54. Mao, J.H., et al. 2005. Genomic instability in radiation-induced mouse lymphoma from p53 heterozygous mice. Oncogene. 24:7924-7934. 